Revue d'histoire de l'Amérique française

ZAS REVUE D.HISTOIRE DE L'AMÉRIQUE FRANÇAISE

\title{
Naissance de la pensée économique au Canada français
}

\section{François-Albert Angers}

Volume 15, numéro 2, septembre 1961

URI : https://id.erudit.org/iderudit/302112ar

DOI : https://doi.org/10.7202/302112ar

Aller au sommaire du numéro

Éditeur(s)

Institut d'histoire de l'Amérique française

ISSN

0035-2357 (imprimé)

1492-1383 (numérique)

Découvrir la revue

Citer cet article

Angers, F.-A. (1961). Naissance de la pensée économique au Canada français. Revue d'histoire de l'Amérique française, 15(2), 204-229.

https://doi.org/10.7202/302112ar d'utilisation que vous pouvez consulter en ligne.

https://apropos.erudit.org/fr/usagers/politique-dutilisation/ 


\section{NAISSANCE DE LA PENSÉE ÉCONOMIQUE AU CANADA FRANÇAIS *}

Ce n'est pas à proprement en historien que je puis m'autoriser à parler de la naissance de la pensée économique au Canada français, mais plutôt en témoin ou en quasi-témoin. J'étais né quand Errol Bouchette, que l'on considère volontiers comme le premier écrivain canadien-français à s'être intéressé un peu profondément aux questions économiques, publiait son Indépendance économique du Canada français. J'ai eu comme professeur, et je suis honoré de pouvoir dire même comme maître, celui qui a pris chez Errol Bouchette le goût d'être notre premier économiste: Édouard Montpetit. Et je suis à vrai dire le seul économiste professionnel de ma génération; c'est-à-dire le seul de mon âge qui ait eu — non pas des idées sur les problèmes économiques - mais qui ait consacré sa vie à l'étude et à l'enseignement de l'économique.

Autrement dit, en soixante ans, ou quatre générations de quinze ans, le Canada français n'a eu, à chaque étape, qu'un seul spécialiste des questions économiques: Errol Bouchette, Édouard Montpetit, François Vézina et moi-même. Je n'ai pas intercalé le nom d'Esdras Minville, de la même génération que François Vézina, parce qu'il s'est toujours lui-même considéré, plus comme un sociologue ou un philosophe social bien au courant des questions économiques, que comme un véritable économiste. A vrai dire cependant, plus que Montpetit encore qui s'est engagé dans une voie plus scientifique, il a été le véritable continuateur d'Errol Bouchette. Car ce dernier non plus n'a pas, à proprement parler, été un économiste; mais bien plutôt un essayiste politique, qui utilisait des connaissances économiques bien établies. Une fois parti dans cette voie, il aurait fallu tenir compte aussi

* Causerie prononcée au dîner de l'Institut d'histoire de l'Amérique française, le 8 avril 1961. 
d'Olivar Asselin (génération de Montpetit) et de Victor Barbeau (génération de Vézina) ainsi que, dans ma génération, de JeanMarie Nadeau. De toute façon, c'est dans la génération qui suit la mienne que commence véritablement la multiplication, maintenant en progrès, du nombre de Canadiens français prêts à envisager la spécialisation dans la carrière de l'économique.

Quant à ma justification de dépasser le rôle du pur témoin pour esquisser un peu d'histoire, je pourrais sans doute la trouver dans le fait que nos historiens professionnels n'ont abordé que d'assez loin notre histoire économique; et encore moins celle de notre pensée économique, qui reste fort mal connue. Ce n'est d'ailleurs pas chose facile. L'historien trouve sans doute aventureux l'économiste qui se mêle d'histoire; mais l'économiste manque rarement de relever des naïvetés scientifiques chez l'historien qui n'est pas aussi un économiste. Il y a là un double genre plein de périls pour quiconque ne maîtrise pas les deux disciplines.

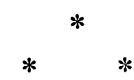

Ces considérations nous invitent d'ailleurs à méditer sur les divers sens du thème lui-même: la pensée économique. En fait celle-ci est comme la vie économique: elle n'attend pas les économistes pour exister. En ce sens, il y a toujours une pensée économique; et il y en a toujours eu nécessairement une au Canada français, parce qu'il y a toujours eu des individus et des gouvernements qui ont dû orienter leurs réalisations respectives dans un sens ou dans un autre. L'idée que la pensée économique a pu ne pas exister chez les Canadiens français, puis apparaître tout à coup ou progressivement, se réfère donc à quelque chose de particulier: à l'idée d'une pensée scientifique, d'une prise de conscience ordonnée, synthétique, rationnelle.

A la vérité, étudier la pensée économique d'un peuple, ce n'est donc pas, ça ne peut pas être, considérer exclusivement ce qu'ont pensé et écrit, ou ce que n'ont pas pensé et pas écrit des économistes ou même des essayistes. C'est étudier aussi l'histoire des faits économiques et en dégager le sens, en démêler les déci- 
sions conscientes des enchaînements fatals. Par suite, quand on a développé au Canada français la thèse de l'absence de pensée économique pour expliquer notre infériorité économique, on a voulu dire que la quasi absence d'économistes et le peu d'intérêt accordé à la science économique par la population et ses élites, expliqueraient les mauvaises orientations de nos réalisations économiques. C'est très honorable pour nous, économistes, mais en toute honnêteté je serai obligé de me demander si telle est bien la vérité, et de n'être pas très catégorique dans mes conclusions. Cette thèse s'est d'ailleurs précisée en une hypothèse, tout de suite acceptée par plusieurs comme une certitude, sur la foi de sa vraisemblance: c'est pour avoir ignoré les impératifs de la science économique que nous serions tombés dans l'agriculturisme et l'antiétatisme, causes formelles de nos difficultés.

Le malheur, c'est que cette thèse contient trop de vrai pour être complètement démolie comme étant totalement fausse; et trop de faussetés pour être considérée comme suffisamment exacte, et par conséquent, valable. Pour qu'elle soit démontrée, il faudrait non seulement prouver qu'il y a eu véritablement une pensée agriculturiste; mais aussi bien que cette pensée a vraiment conditionné toute notre politique, empêché notre société de sécréter les éléments nécessaires à son développement, et privé particulièrement la société d'hommes d'affaires et engendré une politique agriculturiste.

Je ne m'engagerai pas sur la première de ces deux voies qui relève davantage du travail historique pur. Il est d'ailleurs indiscutable, pour tous les témoins du dernier demi-siècle, qu'il y a eu, dans tout un secteur de notre population, autour des hommes d'Église en particulier, un fort attachement à la vie rurale et une forte propension à dénoncer les dangers de l'industrialisation et de l'urbanisation. Mais cela a-t-il dépassé au Canada français ce qu'on trouve partout ailleurs, et notamment dans les encycliques papales elles-mêmes ? Ces mises en garde morales ont-elles vraiment dominé la pensée de ceux qui ont dirigé notre politique, et conditionné leur action au point de leur imposer l'abstention de participer au mouvement d'industrialisation, qui nous aurait ainsi échappé ? Tout ce qu'on peut 
dire pour le moment, c'est que ces thèses ont été appuyées sur des indications ou des citations fort générales ou fort partielles, dont on a déjà commencé à démontrer qu'elles faussaient la pensée même de leurs auteurs. ${ }^{1}$

Quant aux faits, nul ne peut nier que la société canadiennefrançaise est devenue, après la Conquête, une société rurale. Est-ce l'agriculturisme qui en a été la cause, ou la décapitation de notre élite et l'occupation forcée des postes économiques par le conquérant? Une fois les effets de la conquête consommés, cette société rurale ne pouvait pas aisément produire des hommes capables d'aborder l'économique avec de longues et fortes traditions d'affaire. Il fallait sûrement compter ensuite sur un long processus d'évolution pour y arriver. Dans de telles conditions, est-ce bien l'agriculturisme qui va consacrer l'hégémonie des Britanniques dans les développements en cours ? Ne serait-ce pas plutôt la difficulté sinon l'impossibilité, pour la collectivité canadienne-française, de rivaliser, face d'ailleurs à un gouvernement hostile, avec des hommes d'affaires anglophones alimen'és en fonds et en traditions par le personnel et l'argent de la métropole?

A un moment donné, les Canadiens français, coincés par le blocage des terres, devront émigrer en masse vers les États-Unis. Une pensée économique rationnelle aurait vu la solution du problème dans l'industrialisation. Et au besoin, devant la carence d'entrepreneurs canadiens-français, par le truchement d'une politique à forte saveur étatique. Est-ce bien par agriculturisme et par antiétatisme que cela ne s'est pas fait ? Ne serait-ce pas surtout parce que le gouvernement, sous le contrôle de la Métropole, accepte une politique coloniale qui rend l'industrialisation impossible et qui favorise, de toute façon, les éléments oligarchiques anglophones?

Peut-on même constater au moment critique de l'industrialisation, cette carence d'hommes d'affaires canadiens-français de grand calibre, carence qui donnerait du corps à la thèse agriculturiste? Cela ne semble pas très évident, car les Canadiens

1 Dominique Beaudin, «L'agriculturisme, margarine de l'histoire »,
L'Action Nationale, mars 1960. 
français paraissent au contraire faire assez bonne figure et améliorer considérablement leur situation entre 1875 et 1910.

Ce que l'historien peut manquer ici de voir, quand il n'est pas suffisamment familier avec le fonctionnement d'une économie de libre entreprise, c'est que l'entrepreneur, le grand entrepreneur surtout, est un phénomène d'exception, qui n'a pas nécessairement besoin d'être en plein accord avec les normes éthiques de la société dans laquelle il surgit. En fait, il en est souvent le mouton noir, ce qui ne l'empêche pas de s'enrichir et de créer. C'est autre chose que d'inciter le Canada français, placé dans une situation critique, à être un peuple entier de surhommes héroïques et parfaitement lucides, parce que cela lui serait nécessaire pour dominer rapidement une situation; et autre chose, que de faire l'histoire en attribuant à l'absence de cette anormalité, la cause de ses difficultés.

En lisant l'Histoire de la province de Québec de Rumilly, on perçoit au contraire que l'évolution du Canada français sur le plan économique paraît se dérouler assez normalement au début du $X X X^{\mathrm{e}}$ siècle, sans souffrir d'un complexe comme celui de l'agriculturisme. Dès 1875, notre société produit ce qui a été, apparemment, notre premier grand homme d'affaires: LouisAdélard Senécal. Il est donné à ce moment comme l'égal, le pair de Sir Hugh Allan, l'homme du Pacifique Canadien. ${ }^{2}$ D'autres, nous dit Rumilly, ont déjà réussi dans le genre ancien: Joseph Cauchon, Joseph Shehyn, Pierre Garneau, Isidore Thibaudeau; mais Senécal est du type moderne, le type du spéculateur audacieux. $^{3}$

Il est d'abord le propriétaire du chemin de fer RichelieuDrummond-Arthabaska; puis du chemin de fer Québec-Montréal, en même temps que directeur général du chemin de fer du gouvernement provincial Montréal-Ottawa. ${ }^{4}$ Il organise la navigation à vapeur sur la Saint-François et la Yamaska; établit d'importantes scieries à Pierreville; organise à Londres la com-

2 Robert Rumilly, Histoire de la Province de Québec, II: 21.

3 Ibid., III : 21.

4 Ibid., II : 21; III : 21, 158-180. 
pagnie pour la pose du câble transatlantique de Halifax à la côte anglaise; négocie à Paris l'organisation d'une vaste compagnie pour l'exploitation des ressources agricoles, forestières et minières du Québec. ${ }^{5}$ De 1879 à 1882, on évalue ses bénéfices nets à quelque $\$ 400,000 .^{6}$ En 1883 , il achète un moulin à Montmorency, une forge à Radnor, des limites à bois et des terres, par une transaction qui met en jeu $\$ 1,600,000$., « la plus forte du genre jamais conclue par un particulier dans le Québec $\gg ;^{7}$ et la semaine suivante, il pouvait remettre un chèque de $\$ 100,000$. en dot à sa fille, qu'il marie. Il sera le principal bailleur de fonds de «La Presse ». ${ }^{8}$ Quand il meurt, en 1888, il est considéré comme l'homme le plus riche de Montréal. Mais on commence à dire qu'il se fait dépasser par les Van Horne et les Donald Smith du Pacifique. Ceux-ci gagnent du terrain parce que leurs entreprises sont d'envergure nationale et rapportent de plus grosses sommes. ${ }^{9}$

Premier trait qui a beaucoup plus de sens pratique que l'agriculturisme pour expliquer l'écart qui tendra quasi fatalement, si rien de spécial n'intervient, à assurer le progrès plus rapide des intérêts anglo-canadiens que celui des intérêts canadiens-français, d'ailleurs appuyés sur une organisation beaucoup plus précaire. La réserve québecoise, en enfermant nos hommes d'affaires dans le Québec, en assurant aux AngloCanadiens la possibilité de plus fortes accumulations de capitaux à l'échelle nationale, joue contre nous au départ de cette course pour la maîtrise économique de notre propre province dans les cadres de la Confédération.

A tout événement, au cours des années qui suivent, les Canadiens français semblent tenir assez bien leur place dans l'évolution économique de la Province. Plusieurs noms d'industriels créateurs viennent s'ajouter à celui de Senécal: Guillaume Boivin dans la chaussure; Joseph Barsalou dans les savons; F.-L. Béique aux débuts de notre industrie hydro-électrique

5 Ibid., III : 21; IV : 49.

6 Ibid., III: 66.

7 Ibid., IV : 69.

8 Ibid., V: 92.

9 Ibid., V: 263. 
(Royal Electric et Chambly Manufacturing) ; Alfred Dubuc, le grand rival des Price, dans la pâte à papier; Joël Leduc, dans les scieries; Édouard-Alfred Lacroix, qui fonde la North Shore Power et établit la première ligne de transmission dans l'Empire britannique; J.-B. Rolland dans le papier, à Saint-Jérôme; et finalement, au début du $\mathrm{XX}^{\mathrm{e}}$ siècle, le plus grand de tous, LouisJoseph Forget, et son neveu Rodolphe. ${ }^{10}$

Forget est, à ce moment, nous dit Rumilly, le plus grand agent de change et le plus grand brasseur d'affaires de la rue Saint-Jacques. Il est l'âme de tous les grands mergers qui se forment à l'époque: merger de l'électricité et des services publics, merger des textiles, merger de l'acier, merger du ciment, «contrôle » de la compagnie Richelieu, ancêtre de la Canada Steamships, financement de la Wayagamack. Il finit même par devenir assez grand pour être admis dans le Saint des saints: le conseil d'administration du Pacifique Canadien ! C'est le génie financier du siècle.

Son éviction de tous ses intérêts par ses associés anglocanadiens, quand il sera vieilli et usé, vers 1910 , et le retrait de son neveu vers Québec où il forme un merger secondaire de l'électricité et des services publics, marquent la fin de la poussée canadienne-française.11 De 1910 jusqu'à la guerre de 1914, des noms de Canadiens français sont associés à l'administration des grandes affaires qui se forment pour l'exploitation de nos ressources naturelles. Mais ce ne sont plus des noms d'hommes d'affaires: ce sont des noms de ministres du cabinet provincial, que des capitalistes anglo-canadiens et surtout américains invitent à leurs conseils d'administration, en témoignage ... disons, pudiquement, de gratitude! Est-ce vraiment l'agriculturisme qui explique ce recul ? Ne serait-ce pas plutôt la force irrésistible, pour un petit peuple encore à ses premiers pas, de la marée de capitaux britanniques et surtout américains qui inonde la province? Et ne serait-on pas simpliste d'appeler antiétatisme, la

10 Ibid., III: 107 et 126; VI : 310 ; VII : 241; VIII : 313.

11 Ibid., VI: 310 ; VII: 78-79; VIII : 68-69, 173; IX: 142-143, 267-269; $\mathrm{X}: 26$ et 150 ; XI: $103-104,139,174 ; \mathrm{XII}: 62-65$; XIII: 56 ; XV: $34-39$; $\mathrm{XVI}: 72$. 
condescendance de nos hommes d'État envers ces grands capitalistes?

D'ailleurs, ces hommes d'Etat sont-ils agriculturistes ? N'en ont-ils que pour l'agriculture et la colonisation et méprisent-ils l'industrie ? Bien au contraire ! Dès le dernier quart du XIX ${ }^{\mathbf{e}}$ siècle, c'est-à-dire donc dès que l'industrialisation du Canada devient possible, nos gouvernements provinciaux favorisent par tous les moyens qui leur paraissent possibles, l'industrialisation du Québec. Au point même de donner pratiquement nos ressources aux capitalistes étrangers, de crainte que de trop grandes exigences de notre part ne les détournent de la Province. Des hommes comme Parent et Gouin ont décidément une pensée économique: ils veulent industrialiser le Québec. Ils ont même tellement la pensée économique de leur temps, en terme scientifique, c'est-à-dire le libéralisme économique, qu'il n'est pas sûr que la présence de plus d'économistes professionnels chez nous aurait, à ce moment, vraiment amélioré la situation en termes d'économie nationale canadienne-française. Bien loin de là !

Le point tournant de notre histoire économique, et qui condamnait d'avance un Forget à la dépossession ultime, je le vois se produire en 1897. C'est un Canadien français qui, à ce moment, aurait songé à mettre en valeur les chutes de Shawinigan et qui en demande la concession. ${ }^{12}$ Il s'appelait Uldéric Carignan, et son associé Navigius Malhiot. C'est finalement le groupe HoltRussell qui l'obtient et forme la Shawinigan Power. Pourquoi ? Carignan, dit-on, ne pourra compter que sur l'argent des épargnants; alors que le groupe de la Shawinigan, composé de véritables capitalistes, offre des garanties plus certaines de succès! Cette réaction, qui est dans la plus pure tradition de l'économie libérale du temps, un économiste pur de l'époque l'aurait sans doute appuyée de toute la force de ses convictions scientifiques. Ce qui a fait défaut à ce moment, en somme crucial de notre histoire économique, ce n'est pas la domination d'un sentiment agriculturiste, ni même l'absence de pensée économique scientifique, c'est l'absence d'une pensée nationale, qui aurait

${ }^{12}$ Robert Rumilly, ibid., v : 14. 
suggéré au gouvernement une politique d'appui et de soutien à des hommes d'affaires canadiens-français.

Or c'est justement ce qu'écrira Ėrrol Bouchette quelques années après l'incident. Il pourra l'écrire probablement en partie parce que tout en connaissant l'économie, il n'est pas économiste, donc moins intéressé à la théorie pure de son temps qu'aux exigences d'une pensée politique. De sorte qu'il fait figure de précurseur, non seulement en tant qu'il annonce le développement au Canada français d'un intérêt pour la science économique envisagée sur une base professionnelle, mais aussi en tant qu'il échappe au courant de pensée libérale de l'époque et qu'il nous lance déjà dans la voie d'une économie destinée à favoriser une politique économique consciente. Mais avant Bouchette, Etienne Parent avait parlé d'économie dès 1846. Dans quelle circonstance et de quelle façon? Voyons un peu.

L'Angleterre venait d'abandonner les Corn Laws, c'est-à-dire d'accepter pratiquement de s'abandonner au libéralisme intégral. En conséquence, les colonies britanniques perdent leurs privilèges coloniaux sur le marché de Londres; et avec la fin de la politique coloniale, on peut prévoir un développement économique autonome des colonies. Parent, qui a lu les meilleurs économistes du temps, voit que cela va signifier éventuellement un développement industriel canadien. Il nous supplie, au nom de l'intérêt national, de nous intéresser à l'industrie et d'étudier l'économie politique.

Les textes de Parent ${ }^{13}$ témoignent en fait, incidemment, des progrès de notre nationalité, déjà à ce moment-là. 1840 paraît être, d'après lui, la période où nos commerçants prennent de l'importance. Il parle, au sujet de la «classe des marchands canadiens $»(.$.$) , « d'un essor tout à fait encourageant pour les$ autres branches d'industrie ». Ils cessent, nous dit-il, d'être «les agents secondaires des marchands bretons », (...), «ils s'éman-

13 Etienne Parent, Discours devant l'Institut Canadien, (Lowell, Gibson, Montréal, 1850). 
cipent de cette tutelle peu honorable et peu profitable à la fois $»{ }^{14}$ pour traiter directement avec les manufacturiers et les marchands des Îles Britanniques. Il parle aussi de «nos industriels aisés».

Mais ce qui l'inquiète, c'est que ceux des nôtres qui ont atteint ce niveau ne sont ni aussi instruits, ni aussi riches que leurs concurrents. Et qu'ayant le privilège de l'aisance, qui leur permet de faire instruire leurs enfants, ils songent plus à les diriger vers les professions libérales que vers la perpétuation de leur commerce ou de leur industrie. Parent déplore qu'ils ne se montrent également ni aussi agressifs, ni aussi progressifs que les hommes d'affaires anglo-canadiens. Bref ils n'ont ni l'expérience, ni la tradition des autres. Et au surplus, ils ne semblent pas donner à l'industrie «la considération qu'elle devrait avoir dans l'intérêt de notre nationalité ».

Est-ce à un excès d'engouement pour l'agriculture, à la domination de notre société par un sentiment agriculturiste qu'il attribue le fait ? Non ! mais à ce fait que les nouveaux bourgeois prennent modèle sur nos vieilles familles historiques de noblesse, qui conservent les préjugés de leur classe pour le travail des mains et le négoce; et qu'ils voient dans l'accès aux professions libérales une façon d'ennoblir leurs enfants. ${ }^{15}$

Or l'industrie s'en vient chez nous. Et si ce n'est pas nous qui la développons, ce seront les autres. «Veut-on pas se laisser déborder, absorber, écraser par les autres nations, qu'on fasse comme elles; qu'on travaille avec ardeur, avec intelligence, avec constance comme elles. Les nations lâches et abruties étaient autrefois la proie des nations guerrières; maintenant les peuples indolants et ignorants seront exploités par les peuples industrieux et intelligents. ${ }^{16}$ Voilà ce que nous dit Parent. Et encore : «En vain nous retrancherions-nous derrière des traités; en vain nous ferions-nous un rempart de tous les principes de la morale publique, du droit naturel et du droit des gens; il est un droit qui dans le monde et surtout entre peuples, l'a presque toujours emporté sur tous les autres droits, et ce droit est celui du plus

14 Op. cit., 11-12.

15 Ibid., 8.

16 Ibid., 30. 
fort ?» ${ }^{17}$ Le plus fort en industrie comme en armes ! Car « une nationalité, pour se maintenir doit avoir pour point d'appui des hommes réunis en société, et ces hommes doivent posséder une importance sociale égale, pour le moins, à toute force dénationalisatrice qui agit soit du dedans, soit du dehors. Or, qui fait la puissance sociale surtout en Amérique ? Il n'y a pas à s'y méprendre, c'est l'industrie. ${ }^{18}$ D'où la conclusion déjà formulée par Parent au départ de son argumentation: "Si nous voulons conserver notre nationalité, il faudra nous assurer une puissance sociale égale, pour le moins, à celle qui lui sera opposée.»19

$\mathrm{Si}$ nous refusons de nous en rendre compte, un historien écrira un jour ceci des Canadiens français :

Si l'on en croit les mémoires du temps, la principale cause de décadence d'un peuple aussi intéressant (que le peuple canadien-français) fut l'éloignement des classes aisées, les seules qui pussent se procurer de l'éducation alors, pour toute espèce d'industrie. Cela se conçoit, en effet, dans un pays où l'industrie était la seule source de richesse, et où la richesse était le plus grand sinon le seul moyen d'acquérir de l'importance sociale. La masse du peuple dut être livrée à l'influence et à l'action dénationalisatrice des chefs d'industrie de la race rivale, et perdre ainsi avec le temps son caractère national. ${ }^{20}$

Donc aller vers l'industrie, mais aussi étudier, étudier l'économie politique. Pourquoi ? Sans doute parce que c'est la science nouvelle qui éclaire l'avenir. La science qui empêche les faux pas dans le développement économique. Mais aussi, quant à nous, pour un motif plus spécial. Nous allons être aux prises avec " une race d'hommes qui semble avoir entrepris la conquête ou la rénovation du monde par l'intérêt matériel. Son Dieu: c'est Plutus; ses enfants ne naissent, ne vivent que pour le gain; pour eux, il n'y a d'autres rêves que des rêves de fortune rapide et colossale, pour eux point d'aurea mediocritas. » 21

\footnotetext{
17 Ibid., 8.

18 Ibid., 11.

19 Ibid., 11.

20 Ibid., 19.

21 Ibid., 30-31.
} 
Faudra-t-il que nous partagions aussi, pour les égaler, « cette avidité d'acquérir, cet excès d'acquisivité », qui «doit souvent porter à n'être pas trop scrupuleux ». Ce ne serait guère conforme à notre idéal. Ainsi «ceux qui ont à traiter d'intérêts communs avec des gens qui ont ce penchant, doivent être en état de faire valoir les arguments et les considérations les plus propres à faire impression sur eux, et à commander leur conviction». L'économie politique nous fournira ces arguments en montrant que des conditions mutuellement avantageuses pour tous, sont celles qui respectent les droits de chacun. «Car, nous dit Say, chez un peuple où l'on se dépouillerait mutuellement, il ne resterait bientôt plus personne à dépouiller. ${ }^{22}$

Pour Parent, la grande solution à tous nos problèmes, c'est l'instruction. Déjà, en 1878, il demande pour le Canada français: «des Écoles pour les Arts mécaniques et les Beaux-Arts, des Écoles pour le Haut Commerce et l'Industrie, des Collèges pour les Belles-Lettres, et des Chaires et Universités pour les Hautes Sciences $\gg .{ }^{23}$

Il y a peu à dire sur les idées d'économiste d'Étienne Parent, en dehors du passage où il salue le génie de Peel, qui a su comprendre, au risque du pouvoir qu'il a effectivement perdu, les grandeurs du libre échange. ${ }^{24}$ Il est surtout intéressant de trouver chez lui tous les thèmes roboratifs que nous employons encore dans nos conférences pour inciter le milieu canadienfrançais à renforcer son effort économique. Nous verrons plus loin que le ton de Bouchette est tout différent et reflète l'état d'une société qui est déjà en marche, alors que Parent parle à une société qu'il faut démarrer. L'impression qui se dégage de Bouchette, c'est que l'invasion du capital américain - et non pas l'agriculturisme - a constitué une nouvelle pierre d'achoppement, une nouvelle conquête; en nous décapitant de nouveau, elle a rejeté la collectivité canadienne-française dans un état qui s'apparente relativement plus, entre 1930 et 1960 , à la situation de 1850, qu'à celle de 1900/1905.

22 Ibid., 32.

23 Discours (Louis Brousseau, Québec, 1878), 122.

24 Discours à l'Institut canadien, op. cit., 34-35. 
Les thèmes de Parent se résument, en effet, comme suit: nécessité de la puissance économique industrielle pour qu'une nationalité puisse survivre et assurer son épanouissement culturel; manque de considération du milieu pour les carrières industrielles; manque de continuité dans la transmission familiale des commerces et des industries; valeur intellectuelle aussi grande et même plus grande des carrières industrielles que des carrières libérales; manque de sens du travail pour la beauté de l'œuvre économique accomplie et, par suite, manque d'un idéal de faire grand et parfait; souci cependant de ne pas copier vulgairement les autres, mais d'adopter des formules d'action accordées à nos traditions.

Dernière question. Parent était-il un isolé, un phénomène, par son intérêt pour les choses économiques? Je n'ai pas de réponse à apporter, car je n'ai pas poursuivi la recherche qui me permettrait de fournir la réponse. Mais j'évoque la question pour au moins signaler que l'entre-les-lignes des discours de Parent nous inciterait à croire le contraire. D'abord, il fait allusion lui-même à un autre personnage qu'il ne nomme pas, mais qui avait nom Amédée Papineau; il le félicite de faire «paraître, dans les colonnes de la Revue Canadienne, des articles (...) pour initier les lecteurs canadiens aux secrets, aux vérités de l'économie politique $\gg .^{25}$ Il fait encore allusion à un enseignement de l'économie politique au Collège de Saint-Hyacinthe. ${ }^{26}$ Il semble donc y avoir un mouvement d'opinion et de curiosité de ce côté, à une époque où la science économique est tout de même relativement très jeune et ne compte guère encore dans le monde entier que quelques grands noms. Avant de trop simplifier sur le thème de l'absence d'une préoccupation de pensée économique au Canada français, il me paraîtrait donc convenable de chercher davantage.

Bouchette aussi lance le mot d'ordre de l'industrialisation. Il inaugure même le slogan «Emparons-nous de l'industrie », en

25 Discours à l'Institut Canadien, op. cit., 24.

26 Ibid., 41. 
parallèle à l'« Emparons-nous du sol », qui avait dominé le siècle précédent. Mais quelle différence de ton entre Bouchette et Parent. Bouchette ne se plaint pas tellement qu'on donne trop d'attention, ni à l'agriculture, ni aux professions libérales. Dans son premier article de 1901, sur L'évolution économique $d u$ Québec, ${ }^{27}$ il constate au contraire "que nous avons accompli beaucoup. En matière commerciale, notamment, malgré notre infériorité apparente, l'histoire dira que nous avons remporté un succès réel en nous assurant même notre faible part, entravés et découragés que nous étions par un groupe d'hommes qui désiraient conserver pour eux seuls un monopole lucratif; et qui y ont réussi pendant quelque temps grâce aux influences puissantes dont ils disposaient en Angleterre, longtemps notre seul, aujourd'hui notre principal marché ».

Il ajoute: «Nous avons longtemps manifesté une tendance trop prononcée vers les professions libérales et la politique. (...) Aujourd'hui cela n'est plus qu'un préjugé qui tend à disparaître, et l'avocat n'est plus le demi-dieu d'autrefois. Nous semblons présentement trop portés vers les positions inférieures, le travail à gages. Il n'y a rien là qui doive surprendre, si nous tenons compte des nombreux obstacles apportés à la colonisation et de l'absence presque absolue d'instruction technique dans notre pays. »

Donc pas véritablement de cri d'alarme. Mais une attitude corroborant pleinement la situation historique qui ressort de l'analyse des faits. Les choses, de leur mouvement naturel, ne vont pas si mal. Ce qui nous menace ce n'est pas l'agriculturisme, l'antiétatisme, mais l'absence d'une politique industrielle nationale face à l'invasion des capitaux américains. C'est la leçon pratique qu'il apporte, dégagée d'une analyse économique des événements et des circonstances dans lesquelles les données économiques sont appliquées au Canada français. A ce titre, Bouchette mérite d'être considéré comme le précurseur de la science économique chez nous. Lui-même ne fait pas d'exposés scienti-

27 Compte rendu de la Société Royale, mai 1901, reproduit dans la Revue Canadienne, août 1902. 
fiques; mais il se sert scientifiquement des connaissances économiques pour en tirer des leçons politiques.

"Il est permis de croire, écrit-il, que nous sommes à la veille de changements économiques. Nous verrons probablement la révolution industrielle prévue et prédite par le prince de Bismarck...» En effet, nos voisins, «les États-Unis d'Amérique, ayant réuni sur leur territoire (...) le surplus de la population de l'univers, ont vu leur puissance industrielle se développer dans des proportions jusqu'ici sans exemple. (...) Les capitaux, les forces industrielles (s'y) concentrent entre les mains d'hommes puissants et audacieux qui ont conçu la pensée de se faire du monde entier leur tributaire. (...) Déjà les trusts, ne trouvant plus de conquêtes à faire chez eux, se préparent à nous envahir ». Que faire ?

La réponse à la question est aussi simple que nette. Il ne s'agit pas de repousser ces forces qui peuvent nous aider à réaliser un développement nécessaire, sinon inévitable. "Mais nous devons, dit-il, les attendre dans une bonne position stratégique afin de rester, quoi qu'il arrive maître chez nous. » Si ce mode d'exposition et ces déclarations avaient été formulés dans l'atmosphère agriculturiste qu'on se plaît parfois à nous dépeindre comme une réalité, Bouchette nous apparaîtrait comme un extraordinaire prophète. Mais en 1901, les Canadiens français, nous l'avons vu, donnent pas mal dans l'industrie. Le gouvernement de Québec, avec S.-N. Parent, est lancé à fond de train dans l'industrialisation. Bouchette ne prophétise pas. Il voit clairement, avec les Américains se pressant à nos portes et l'attitude que prend le gouvernement à leur égard, ce qu'il va advenir de ce premier démarrage canadien-français dans l'industrie. Il ne dit pas, comme Parent, «allons à l'industrie»; mais bien «emparons-nous de l'industrie », c'est-à-dire ayons une politique qui empêchera ceux qui se pressent à nos portes de nous enlever l'industrie.

Il a conscience de lancer son appel à temps, alors que «les troubles économiques qui existent ailleurs ne nous ont pas encore atteints ». Par suite, insiste-t-il, nous pouvons, «nous avons le 
devoir de profiter de ce répit pour chercher d'avance une solution et faire en sorte que la grande industrie, en s'implantant dans notre pays, n'asservisse pas notre peuple...»».

Où éclate la connaissance de l'économique chez Bouchette, par opposition par exemple à un Bourassa qui au même moment nous recommandait de ne pas viser plus haut que la petite et la moyenne industrie, c'est la façon dont il a compris que c'est au niveau de la grande industrie que se joue la partie. D'où au contraire d'un Bourassa, devant la même constatation de notre faiblesse, il conclura à la nécessité d'une politique gouvernementale qui nous permet d'accéder à la grande industrie et de la garder.

La concentration, nous expose-t-il, dans une série d'articles ultérieurs, dans la Revue Canadienne de 1905, est la loi du progrès économique. ${ }^{28}$ Il ne s'agit pas de la dénoncer, car elle « est une application nouvelle des principes de l'organisation et de la division du travail». Cependant, continue-t-il, «il faut s'entendre sur la manière de l'appliquer ». La concentration industrielle sous la forme bienfaisante n'est pas le trust américain. «Celle-ci en général est un monopole abusif et dangereux qui a surgi sous la pression de la nécessité (...) Il faut donc que nous opposions à la nouvelle méthode industrielle qui nous arrive sous la forme du trust, une méthode mieux conçue, moins dangereuse et qui nous fournira en aussi grande abondance ce capital médiat dont nous avons besoin. »

Il faut donc une politique. "De nos jours, écrit-il en 1901, pour implanter dans un pays la grande industrie, il faut de toute nécessité une organisation puissante, appuyée d'une politique industrielle de la part des pouvoirs publics. » Et en 1905, il précise: «Nous croyons pouvoir affirmer que, sans le développement industriel l'augmentation numérique des Canadiens français deviendra de moins en moins sensible (...). Il est donc évident pour nous que si le Canada français veut vivre, il doit se développer par l'industrie comme par l'agriculture. Pour que son cœur

28 Cette série d'articles a d'abord été publiée en volume dès 1905 , sous le titre de Emparons-nous de l'industrie, puis rééditée ultérieurement sous le titre de L'indépendance économique du Canada français. 
batte avec force, il lui faut remplir d'air ses poumons. Et ce cœur, au Canada français du moins, n'est-ce pas le corps législatif et gouvernant, la seule législature française de toute l'Amérique, dont les pulsations doivent alimenter les artères d'un sang abondant, pur et généreux ? N'est-il pas évident que ce cœur bat trop faiblement ?»

Quelle est la politique de Bouchette ? Elle se présente, dans ses grandes lignes, en deux parties et se ramifie ensuite en des politiques de détail. Les deux grandes orientations: développer un enseignement technique complet et parfaitement coordonné; et instituer un prêt industriel de préférence non gouvernemental, mais appuyé par l'État, afin de permettre aux nôtres de développer eux-mêmes les industries basées sur les ressources naturelles, spécialement sous la forme de syndicats communaux dans le cas de l'exploitation forestière. Par le détail, la politique de Bouchette concerne surtout les règles en fonction desquelles devraient être accordées les concessions forestières et les chutes d'eau, ainsi que celles qui devraient conditionner l'exploitation forestière. Il demande l'abandon de la vente des ressources et la substitution du bail emphytéotique, ainsi que l'exploitation rationnelle des forêts.

Même si l'on a en quelque sorte entendu Bouchette, lorsqu'on a fondé des écoles techniques et l'École des Hautes Études commerciales, que ne l'a-t-on mieux écouté quand il insistait sur le fait que le système d'enseignement technique veut dire quelque chose d'« organisé, indiquant une chose complète où tout s'emboîte et se tient », sans quoi, avertissait-il, on aboutirait à « des efforts stériles ou donnant lieu tout au moins à un grand gaspillage de forces ». Que n'a-t-on plus tôt créé ce prêt industriel, qui n'existe d'ailleurs pas vraiment encore, et en vertu duquel le gouvernement de la Province aurait pu éviter la répétition d'incidents comme celui de Carignan et des chutes de Shawinigan, qui se sont depuis répétés à l'infini ?

En 1905 cependant, Bouchette n'est plus tout à fait aussi serein qu'en 1901. Il voit sans doute se dessiner le mouvement d'invasion qu'il annonçait en 1901 et qui effectivement bat alors son plein. Il avertit que «le Canada français (...) se conges- 
tionne et s'étiole derrière une muraille de Chine que seule la grande industrie, dirigée dans des voies naturelles, pourra renverser »; que «le groupe français du Canada, malgré ses qualités physiques et intellectuelles est (tombé) économiquement, au dernier rang des groupes canadiens ». Il annonce que pour le Canada français c'est le début d'une ère au cours de laquelle «la question économique deviendra plus que jamais une question nationale » et qu' « elle restera, pour ainsi dire, la seule question jusqu'à ce qu'elle soit résolue ». Il se plaint que la masse des Canadiens français se complaît trop dans ses succès démographiques, montre une largeur insuffisante de vues dans les conceptions économiques et ne comprend pas assez l'importance du développement industriel. Son texte démontre même ce que l'on sait déjà: l'existence d'esprits craintifs devant l'orientation qu'il donne.

Qu'ils nous disent, ceux-là, écrit-il, depuis quand la victoire et la puissance ont détruit chez les peuples les glorieuses traditions? Depuis quand la défaite et la servitude développent chez eux les qualités nobles et viriles ? Sont-ce les faibles qui dirigent les forts, les esclaves qui commandent aux maîtres ? Qu'adviendrait-il, si par malheur il nous arrivait d'avoir à subir la loi du vainqueur économique, le plus terrible de tous; si en abdiquant virtuellement notre influence et nos droits, nos ouvriers devenant des ilotes, nos agriculteurs des paysans ruinés, nos classes instruites ou prétendues telles, des prolétaires - comme le sont déjà les trois quarts de nos médecins, avocats et fonctionnaires ainsi que la presque totalité de nos instituteurs ? Est-ce quand tout cela serait consommé que nous pourrions prétendre prêcher sur ce continent, comme le firent nos pères, la sainte croisade de la vérité, de la justice et de la liberté ? Pourrions-nous espérer qu'en de telles conditions notre population s'accroîtrait et que nous fonderions des familles saines et nombreuses ? Verrait-on fleurir dans un pareil milieu l'agriculture, les lettres, les sciences, les arts, sans parler de la morale et de la religion ! (...)

Ah ! ne nous y trompons pas. Nous n'accomplirons nos destinées qu'à la condition d'être de toutes 
manières les forts de notre siècle. Nous n'y arriverons que par un effort constant et bien dirigé; par la résolution inébranlable de mettre en honneur et en pratique parmi les nôtres cette science «qui constate (et qui applique) les lois générales déterminant l'activité et l'efficacité des efforts humains pour la production et la jouissance des différents biens que la nature n'accorde pas spontanément et gratuitement à l'homme ».

On peut trouver ici l'indice qu'il y avait peut-être chez nous cette sorte d'hommes qu'on a appelés des agriculturistes, bien que - nous l'avons précédemment souligné - il aurait fallu, avant de lancer la formule, l'appuyer sur autre chose que des bouts de textes, par trop épars. De là à conclure que ce sont ces points de vue qui ont réellement causé premièrement et principalement notre infériorité économique, il y a tout de même à prendre garde. Encore une fois, la pensée de Bouchette elle-même, placée en relief des événements, nous montre, une fois de ọlus, qu'à ce moment, le problème réel n'a été dans les mains ni de la masse de la population ni des élites pro-rurales, mais bien d'une série de gouvernements réellement partisans de l'industrialisation. Malheureusement, ils étaient dépourvus de toute pensée nationale. Sans quoi ils eussent accepté des formules de développement que Bouchette lui-même leur proposait afin de garder le contrôle aux mains des Canadiens français. Les contemporains comme Bourassa, Asselin, Groulx, Montpetit, Minville, etc., qui dénonceront plutôt la politique des grands partis traditionnels à l'échelle nationale, la politique des «vieux partis», et la partisannerie politique des Canadiens français, paraissent plus près de la vérité que la thèse agriculturiste, en dépit de toutes ses vraisemblances.

Il semble assez clair aussi, encore plus que pour Parent, que Bouchette, pour être probablement le plus lucide de tous, n'était pas un isolé. La Chambre de Commerce de Montréal paraît assez active autour de ces questions; elle trouve des appuis chez des hommes comme Honoré Gervais et d'autres. Si la nécessité d'un enseignement de l'économique et de la préparation aux affaires pour les Canadiens français n'avait pas joui d'un 
degré de reconnaissance suffisamment accentué, comment expliquerait-on le mouvement pour la fondation d'une École des Hautes Études commerciales ?

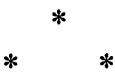

Quoi qu'il en soit, l'appel de Bouchette en faveur de la science économique fit écho dans l'esprit du jeune Édouard Montpetit, avocat et de tempérament plus artiste que rigoureux, mais aidé en cela par la direction de plusieurs de ses protecteurs, qui comprenaient donc eux aussi le problème. Il est assigné, en 1907, au premier cours régulier d'économie politique qui se soit donné au niveau de l'Université, à la Faculté de Droit de Montréal.

Dans ses Souvenirs, M. Montpetit nous rappelle que sa nomination à la Faculté de Droit fut saluée, dans le Canadien, par un journaliste du nom de Gaston Dugas, en des termes qui indiquent que celui-là non plus n'était pas un parfait ignorant en la matière, prêt ou à condamner une initiative étrange ou à faire preuve d'un enthousiasme de néophyte encore mal dégourdi. Il félicite l'Université Laval de la «quasi-création» d'un cours d'économie politique, expression qui annonce le ton du reste. Puis, le journaliste continue:

Je ne connais pas le nouveau titulaire, mais eût-il tous les talents du monde qu'il ne réussirait pas, sans des études profondes, à faire un cours d'économie politique qui exige de l'expérience et de nombreuses recherches. En France, cette nomination ferait sourire. Il faut croire qu'ici on est moins exigeant. (...) Le résultat de cette grave erreur, c'est que ce cours manquant dès en son début d'une interprétation imposante par un savoir et une expérience qui doivent être à la base de toute chaire, ne sera ni plus ni moins qu'une lecture que la grande majorité de ceux qui veulent connaître les principes de cette science préféreront faire à tête reposée dans leur cabinet de travail. Et qui pourra les en blâmer ?

Rappelons que les seuls titres de M. Montpetit à un tel cours, à ce moment, était sa licence en droit sans formation 
économique, et la rédaction de deux conférences reproduites dans la Revue Canadienne. Frappé par cette remarque dure mais juste, M. Montpetit demanda et obtint d'aller faire un séjour d'études en France. C'est l'École des Hautes Études commerciales, tout juste fondée, qui eut l'honneur de patronner son voyage. Elle aura ainsi donné à la province de Québec son premier économiste vraiment formé par des études régulières et vraiment consacré à l'enseignement des sciences économiques.

L'œuvre d'Édouard Montpetit chez nous a été une œuvre immense, quoi qu'en disent parfois ceux qui jugent d'une carrière économique à la pesanteur du papier imprimé et au nombre d'équations ou d'intégrales qui s'y trouvent imprimées. Sans doute, ce ne fut pas une œuvre réellement importante en termes d'ouvrages savants. Mais une œuvre d'économiste apôtre et fondateur, dans un pays où les textes trop savants n'auraient été achetés ni lus par personne. Les conditions dans lesquelles il travaille: multiplicité des cours, même non économiques, secrétariat de la nouvelle université de Montréal, missions gouvernementales et commissions d'enquête, mais plus que tout peut-être, la nécessité de convaincre d'abord le public de l'importance d'une pensée et d'un enseignement économiques, détourneront le professeur d'un travail d'étude en profondeur. Mais il fut exactement l'homme dont nous avions besoin à ce moment, et qui a ouvert la voie à tous ceux qui sont venus depuis.

C'est lui qui crée et qui tient pendant 35 ans la première chaire d'enseignement économique un peu complet au Canada français: celle de l'École des Hautes Études commerciales. C'est lui qui lancera la première Faculté des Sciences sociales, économiques et politiques à l'Université de Montréal; alors école du soir, école de vulgarisation plus que de véritable standing universitaire, mais qui, dans l'esprit même de son fondateur, devait répandre la bonne parole à qui voudrait l'entendre et préparer le jour où pourrait se constituer un enseignement plus avancé.

Il a installé, au cœur même du Canada français, une conception de la science économique dont l'École des Hautes Études 
garde la marque apparemment indélébile et qui est, en réalité, fort originale et fort prometteuse. Au contact de ses maitres français, c'est surtout au libéralisme économique que Montpetit s'était abreuvé, mais dans un éclectisme de méthode qui excluait les abstractions trop simplificatrices. Par ailleurs, sa formation philosophique thomiste et sa préparation juridique le tiraient en dehors de ce qu'il y avait d'excessivement naturaliste dans le libéralisme intégral. Son adhésion à ce qu'il appelait le catholicisme social libéral en faisait un néo-libéral avant la lettre. Enfin l'influence première de Bouchette combinée avec celle de l'École française le marquait par une adhésion, d'abord à l'idée d'une science économique orientée vers la compréhension des situations concrètes plutôt que vers les jongleries d'abstraction pure ou les uchronies; science ensuite appliquée à promouvoir le mieux-être de la collectivité dans ses dimensions aussi bien nationales que sociales.

On aura beau dire en certains milieux où «scientifique» paraît devoir être synonyme de style revêche ou hermétique, Montpetit fut véritablement économiste, tout en gardant ses allures poétiques et sa liberté de faire des incursions dans bien d'autres domaines. La différence est sensible, par exemple, entre Bouchette et Montpetit. Le premier fait surtout de la politique qu'il appuie sur des considérations économiques, alors que Montpetit expose avant tout des principes et des théories économiques dont il tire ensuite des conclusions politiques au besoin. La manière est donc toute différente. Par ailleurs, le pli que donnera à son esprit un exercice plus poussé et plus systématique de la pensée théorique, le rendra beaucoup moins catégorique dans ses propositions interventionnistes.

Comment concevait-il la science économique ? Très nettement comme une science vraiment expérimentale, en opposition aux tendances abstraites qui ont caractérisé les théories pures des classiques et qui ont été remises à la mode, en ces dernières années, par le keynésianisme et les modèles mathématiques. Fidèle à l'éclectisme de ses mâtres français, mais plus affirmatif que la plupart d'entre eux, Montpetit tient que l'économie politique 
est une science de la vie, qui doit partir des faits vivants, et non pas de postulats, d'abstractions ou de symboles. A son premier cours, il nous lançait toujours cette formule imagée: «L'économie politique s'apprend dans la rue. »

Dans son premier ouvrage, Pour une doctrine, il s'en explique ainsi :

L'économie politique n'est pas une science abstraite, comme on le croit trop. Des traités d'Economique (...) ont l'apparence de manuels d'algèbre. On y a mis force chiffres et d'interminables équations qui chevauchent entre les marges. Les lignes géométriques s'entrecroisent et forment d'étranges arabesques, car on y étudie les courbes et si elles sont concaves ou convexes. (...)

Ce n'est pas le moment de critiquer cette méthode chère à Jevons, Walras, Pareto et autres. Elle présente de l'intérêt. Les chiffres sont positifs ; leur alignement est rigide autant qu'impressionnant. Si le nombre, par une gymnastique sûre, établit la vérité d'une loi économique, il fournit à la science une démonstration indiscutable, un dernier argument et pose l'esprit sur une base inébranlable. Les lois de la vie et de la mort ont été mises en tables. Le hasard même obéit à des principes qui n'ont rien de capricieux et que les géomètres se flattent d'avoir fixés. - Il se peut. (Mais)

(...) l'économie politique est avant tout une science d'observation, très proche de la vie qu'elle s'efforce de pénétrer. Elle tient compte d'abord des faits et, si elle énonce des lois, c'est à la condition de les étayer d'observations répétées.

Il n'en fut pas toujours ainsi. Les premiers économistes ont été trop souvent de purs théoriciens. Ils se tenaient éloignés du monde extérieur, cultivant les idées dans le cabinet de travail. Leur a-t-on assez reproché leur tour d'ivoire et cet homo œconomicus, qu'ils avaient imaginé pour le nourrir de leurs abstractions ! (...)

Ce mépris des faits n'existe plus. (...) la science s'attache au réel. Rien d'autre ne la préoccupe d'abord. Elle part des faits et non plus tant des idées. (...) Par des moyens (appropriés), l'écono- 
miste parviendra à réunir des faits nombreux sur lesquels il appuiera son jugement, sa doctrine. Il pourra dès lors risquer une idée générale et la croire solide scientifiquement. Ces tâtonnements engendreront une certitude au moins relative.

(...) Mais une telle science observe avant de généraliser. C'est une tout autre méthode; et c'est la meilleure.

Encore une fois ce texte n'est pas d'un poète, mais bien d'un économiste. La façon ou la méthode d'approcher la science économique reste encore sensiblement, avec des variantes appropriées au progrès de la recherche en science économique depuis 1926, celles qui prévalent à l'École des Hautes Études commerciales. Et son Institut d'Économie appliquée ne s'en porte pas plus mal.

J'ai d'ailleurs été relativement injuste en laissant entendre que M. Montpetit ne nous a pas laissé une œuvre écrite vraiment importante en science économique. Outre quelques chapitres épars de son cours que l'on retrouve à travers ses volumes où il embrasse d'autres sujets, il a écrit deux ouvrages techniques qui méritent toujours d'être lus et qui sont sans équivalents, par cela au moins qu'ils portent sur des questions canadiennes. Si l'on fait abstraction des articles de revue sur les questions économiques qui se sont considérablement multipliés au Canada français depuis le temps d'Édouard Montpetit, il reste que deux ouvrages techniques achevés et publiés sur des sujets de ce genre constituent encore un record qui a pu être égalé mais, à date, non pas encore dépassé.

Sous le signe de l'or étudie le système monétaire canadien tel qu'il fonctionnait en 1931. L'ouvrage est aujourd'hui périmé à cause des changements nombreux qui se sont produits depuis, mais sa valeur historique est toujours là. Quant Aux Cordons de la Bourse, où l'auteur expose le mécanisme du budget de l'État, c'est un livre encore pleinement valable, d'une qualité exceptionnelle et à peu près unique en son genre. 
Avec Édouard Montpetit, une pensée économique de nature scientifique est donc vraiment née chez nous, en fonction d'une tradition qui nous est en quelque sorte typique et dont l'École des Hautes Études commerciales garde le dépôt. Ce n'est que beaucoup plus tard, au cours de la Deuxième Grande Guerre, que des courants nouveaux, non intégrés à notre tradition propre, s'introduiront d'abord à la Faculté des Sciences Sociales de Québec, qui s'inspirera des traditions keynésiennes pures fort en vogue dans les grandes universités américaines au cours des années '40; puis à la Faculté rénovée des Sciences Sociales de l'Université de Montréal. Un professeur européen, Roger Dehem, y implantera la tradition austro-saxonne de l'économie pure. Mais il faut bien se garder de conclure de là, à l'exactitude de la thèse qui prétend attribuer notre infériorité économique à ce retard dans le développement intensif de la pensée et de la recherche économique chez nous. Dans la mesure même où c'est l'atmosphère générale, le climat social de la Province qui est en jeu; dans la mesure même du manque de largeur de vue dans les conceptions économiques de notre population en général et de nos hommes d'affaires en particulier, ce dont Bouchette se plaignait au début du siècle, il n'y a pas de doute que la thèse contient une part de vrai. Dans la mesure au contraire où l'on songe aux conseils que des économistes avertis auraient pu donner au gouvernement de la province de Québec, la situation est beaucoup plus complexe. Qu'était-ce, en effet, et que pensait un économiste averti en 1900 ou en 1915 ? N'aurait-il pas en définitive conseillé au gouvernement de confier l'administration de nos ressources naturelles au plus offrant et au plus puissant? Et alors?

Comme il semble bien que ce soient vraiment les gouvernements qui ont tenu notre destinée économique entre leurs mains au moment crucial de notre histoire, celui de notre industrialisation, prenons garde de verser dans l'anachronisme et de faire l'histoire en fonction des ressources et des possibilités qu'offre aujourd'hui une science, la science économique. Cette science n'en était encore, au début du siècle, qu'à sa toute première enfance, qu'à ses premiers balbutiements. A cause de 
cela, elle n'a pu, à cette époque, et dans tous les pays, acquérir d'influence que dans la mesure où elle a pu être mise au service d'intérêts nationaux.

Tout ce que je viens d'écrire n'a, à mon sens, qu'un seul mérite: celui d'être fondé sur des faits plus que sur des hypothèses. Mais des faits fort incomplets, je suis le premier à le concéder. Puisse la controverse qui peut s'amorcer autour de ces idées, orienter nos historiens vers une étude plus approfondie de notre histoire économique, en particulier de celle de la période 1875-1910; et aussi vers une recherche plus exhaustive des expressions d'opinion ou de pensée économique au Canada français depuis la conquête.

FrançoIS-Albert ANGERS 